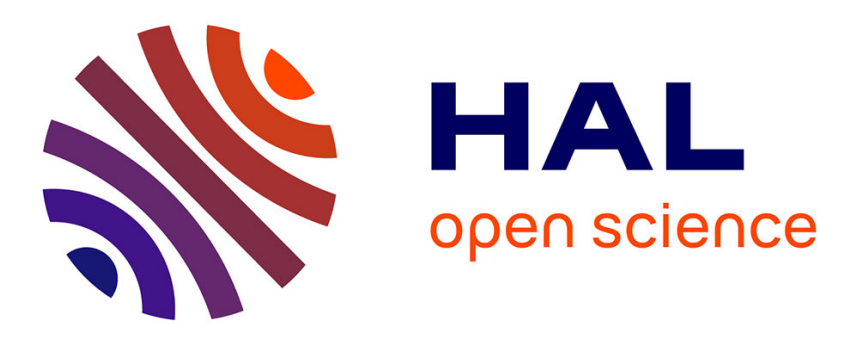

\title{
Generation and stability of cement soap films
}

Djilani Ahmed Abdourahman, Arthur Geniere, Mélodie Auriol, Florent Dalas, Anne-Laure Biance, Marie Le Merrer

\section{To cite this version:}

Djilani Ahmed Abdourahman, Arthur Geniere, Mélodie Auriol, Florent Dalas, Anne-Laure Biance, et al.. Generation and stability of cement soap films. Soft Matter, 2021, 17 (9), pp.2429-2438. 10.1039/D0SM01991G . hal-03166916

\section{HAL Id: hal-03166916 https://hal.science/hal-03166916}

Submitted on 11 Mar 2021

HAL is a multi-disciplinary open access archive for the deposit and dissemination of scientific research documents, whether they are published or not. The documents may come from teaching and research institutions in France or abroad, or from public or private research centers.
L'archive ouverte pluridisciplinaire HAL, est destinée au dépôt et à la diffusion de documents scientifiques de niveau recherche, publiés ou non, émanant des établissements d'enseignement et de recherche français ou étrangers, des laboratoires publics ou privés. 


\title{
Soft Matter
}

\section{ARTICLE TYPE}

\section{Generation and stability of cement soap films ${ }^{\dagger}$}

\author{
Djilani Ahmed Abdourahman, ${ }^{a}$ Arthur Geniere, ${ }^{a}$ Mélodie Auriol, ${ }^{b c}$ Florent Dalas, ${ }^{c}$ Anne- \\ Laure Biance $^{a}$ and Marie Le Merrer ${ }^{* a}$
}

Received Date

Accepted Date

DOI: $10.1039 / x x x x x x x x x x$

www.rsc.org/journalname
Foaming a cementious suspension is a complex process that involves many multiscale chemical, physical and dynamical mechanisms. As a first step, we investigate here experimentally the possibility of withdrawing a single liquid soap film from a suspension of cement. We then determine the film lifetime and if particles are entrained or not. We vary the cement concentration, grain size, rheological properties and withdrawing velocity. We observed that the rheology of the cement paste, characterized through its yield stress, plays a key role in the film formation. We show that an optimum exists, as a low yield stress promotes film creation but is detrimental to the film stability. Another key result is that the rheology alone is not enough to describe film formation: the particle size in the suspension is also crucial, with large particles promoting film creation. Finally, we found that the withdrawing velocity also affects the ability to create films and the possibility to drag particles in them. Experiments performed with a silica suspension for comparison confirm these findings.

\section{Introduction}

Aerated porous solids are interesting materials combining the properties of their solid matrix, such as mechanical properties or fire resistance ${ }^{1}$, to the advantages of air: they are light, have good thermal and phonic insulating properties and reduce the quantity of raw material content, helping industry to address crucial environmental challenges. Besides, they have a porous structure with a large surface to volume ratio. The solid material can be polymeric, metallic ${ }^{2}$ or ceramic. Examples of ceramic foams include materials made from sintered alumina or silica colloids 3 , hydroxyapatite or calcium phosphate foams used as scaffolds for bone replacement applications 55 , and of course cement or concrete foams ${ }^{7}$. Concrete foams allow the building industry to improve the thermal efficiency of building materials, as well as to reduce the environmental footprint due to the production of Portland cement ${ }^{7}$, the most commonly used one, whose composition is presented in the following.

Elaborating a solid cement foam first requires to foam, i.e. incorporate air in, a cement paste. This first step is not as simple

\footnotetext{
${ }^{a}$ Université de Lyon, Université Claude Bernard Lyon 1, CNRS, Institut Lumière Matière, F-69622, Villeurbanne, France. E-mail: marie.le-merrer@univ-lyon1.fr

${ }^{b}$ Centre de Recherche Paul Pascal, UMR 5031, Université de Bordeaux, CNRS, 115 Avenue du Dr A. Schweitzer, 33600 Pessac, France.

${ }^{b}$ LafargeHolcim Innovation Center, 95 rue du Montmurier, 38291 Saint-QuentinFallavier, France.

$\dagger$ Electronic Supplementary Information (ESI) available: Movies 1 and 2 show representative experiments corresponding to the images sequences of Fig. $4 \mathrm{l}$ and 4 , respectively. See accompanying text for details. See DOI: 10.1039/cXsm00000x/
}

as one could expect, because of the properties of the paste. First, the paste is not only a reactive fluid, but it also has a complex rheology, solid-like at low stresses, but able to flow, at stresses larger than a yield stress $\sigma_{Y} \underline{8}$, with a large apparent viscosity. Unfortunately, highly viscous suspensions show lower foamabilities $^{9}$ and the complex rheology (in particular the yield stress) also modifies the stability of the foamed structure $\frac{910}{10}$. Secondly, the paste is also a suspension of particles, typically 1 to $100 \mathrm{mi}$ crons in size, hence comparable to the typical thickness of fresh soap films $11+13$. This then raises new questions on the ability of particles to be trapped in the films. Finally, in order to stabilize the liquid-gas interfaces, surfactants are incorporated in the paste. If they are ionic and have a charge opposite to that of the particles (anionic surfactants for positively charged Portland cement grains), surfactants may adsorb on the particles. A first consequence is that the paste rheology is modified ${ }^{14}$. But this also renders the particles hydrophobic and surface-active, enabling the formation of so-called particle-stabilised foams s $^{915}-17$. This favors the formation of particle-laden films which may ultimately solidify as cement films $s^{18}$ and form closed cell foams with a low thermal conductivity 19 . To summarize, the role of physico-chemical conditions on the fresh foam structure is now understood.

On the contrary, little is known on the dynamical aspects of the foaming of cement pastes, and the foaming of suspensions of particles in general. In the foaming process, the paste and foamed paste undergo strong shear, which might play a key role, in particular by breaking aggregates of particles which might further 
re-assemble in the films 20 . Finally, how dynamical processes and the presence of particles modify film stability remains an open question. Whereas the formation of soap films is known to be governed by surface mechanical properties 21 , which are dramatically modified by the presence of particles 22 , less is known about film stability, which depends on the mechanical deformation or stress $^{23}$ and on the presence of particles that can either inhibit 24 or trigger rupture ${ }^{25}$.

To get some insight on the role of dynamical parameters-shear rates and stresses - on the foamability of fresh cement pastes, we focus here on the particular case of the generation of a single soap film. We investigate the influence of the solid mass fraction, the average particle size and the velocity of film making and determine the ability to generate foam films, whether they contain particles and their stability.

\section{Materials and methods}

\subsection{Samples}

We perform experiments on two different systems: suspensions of non reactive silica beads and reactive fresh cement pastes, composed of cement (Lafarge-Holcim) and of an aqueous surfactant solution. The surfactant nature and concentration are specified below. In some suspensions, a polymeric rheology modifier is also incorporated, as explained below.

The mass fraction of solid material in the cement (resp. silica) suspension, denoted as $w$, is varied in the range $0.5-0.75$ (resp. $0.49-0.83)$. This corresponds to a solid volume fraction in the range $0.24-0.56$ (resp. $0.27-0.66$ ).

On the one hand, the silica particles (Silibeads) are used as received. Their average size varies between 3 and $30 \mu \mathrm{m}$, so they are not colloidal. To probe the effect of particle size, two granulometric distributions have been used, whose characteristics are reported in Table 1. At these mass fractions, the obtained silica suspensions look like pastes.

On the other hand, the cement powder consists in a mix of calcium silicates and aluminates, with a grain size ranging from a few microns to a few tens of microns. In this study, two different cement types are used, whose granulometric properties are summarized in Table 1. Most of the experiments were performed with the product LHY-05381 (CEM I 52.5R from Le Havre Lafarge plant), which we refer to as regular. For a few experiments, a cement with smaller particles (LHY-04190 CEM I 52.5N from Saint-Pierre-la-Cour Lafarge plant), which we refer to as fine, was used. In both cases, the grains have a polyhedral shape. Average grain sizes and standard deviations are then varied by a factor 2 to 4 . These cements are composed of $95 \%$ of clinker (calcium and aluminium silicates) and $5 \%$ of calcium sulfate.

When dispersed in water, the cement particles undergo attractive interactions, mostly due to van der Waals forces and also to ion-ion correlations due to the presence of divalent calcium ions coming from the cement dissolution 26 . Consequently, the fresh dispersion is a paste, i.e. a yield stress fluid, whose yield stress and apparent viscosity increase with the cement content ${ }^{8}$. The fresh paste is always used between 10 and 45 minutes after elaboration, before the acceleration of the setting reaction in the ma- terial (3 hours 27 ) and to avoid significant changes in the paste rheology. This is supported by the fact that we did not observe any time dependency of our measurements.

In order to stabilize the liquid-gas interfaces, as well as to ensure a good capture of the cement particles in the film, an ionic surfactant with an electrical charge opposite to that of the particles is used. For suspensions of negatively charged silica, we use the cationic surfactant TTAB (Tetradecyl Trimethyl Ammonium Bromide). Cement suspensions are composed of various mineral phases whose surface potentials strongly depend on the physicochemistry of the solution which evolves upon time ${ }^{28 \mid 29}$. However, it is well known that anionic additives like superplasticizers or anionic surfactants efficiently adsorb on cement particles to modify their rheology or their contact angle $\mathrm{e}^{14|18| 30}$. We therefore incorporate Sodium Dodecyl Benzene Sulfonate (SDBS), an anionic surfactant, in the cement suspensions. Previous works have shown that the surfactant-induced hydrophobization of particles, which ensures their capture in the soap films, is favored when the surfactant is concentrated enough to adsorb on the solid surfaces, but not too large to prevent surfactant aggregates or bilayers $14[16 \mid 18$. The optimal concentrations were found close to $10 \%$ of the critical micellar concentration $\frac{18}{18}$ so we choose this value in our study. The initial concentration of SDBS in the solution for cement is therefore fixed to $0.167 \mathrm{mM}$, and the concentration of TTAB in the solution for silica is $0.45 \mathrm{mM}$.

Finally, in some experiments (cement samples B and C), we also add to the solution polycarboxylate grafted polyethylene oxide (PCE, Chryso Premia 162) which acts as a polymeric superplasticizer 30 . The polymers are known to graft onto the cement surface and to promote steric repulsion between the otherwise attractive grains. The addition of PCE inhibits the aggregation of the cement particles, and thus results in a viscosity and yield stress decrease, at a given cement content. To ensure that the cement surfaces are well covered by the polymer, the concentration of superplasticizer is chosen to be proportional to the cement concentration and to its specific surface area determined from BET test and reported in table $1^{130}$. The concentration of PCE used is therefore fixed to $0.12 \%$ of the cement mass (resp. $0.44 \%$ ) for the regular cement LHY-05381 (resp. fine cement LHY-04190). When a superplasticizer is used, the cement is first mixed with one half of the water with PCE, before the second half that contains the SDBS surfactant is added.

\subsection{Yield stress measurements}

The rheology of the fresh pastes is that of a yield stress fluid, i.e. they can only flow for shear stresses larger than a yield value $\sigma_{Y}$. We therefore measure the yield stress values for our samples.

In order to assess a large number of formulations with a simple procedure, we used a mini-cone test, a labscale adaptation of the so-called slump test ${ }^{31 / 32}$ as depicted in figure 1, a normalized cone (38 $\mathrm{mm}$ lower and $20 \mathrm{~mm}$ upper diameter, $55 \mathrm{~mm}$ height) deposited on a glass plate is filled with the paste and slowly lifted. Under the action of gravity forces, the paste spreads on the surface and we measure the final diameter $D_{f}$ of the slump. At the end of the process, gravitational pressure is balanced by the yield 


\begin{tabular}{|c|c|c|c|c|c|c|c|}
\hline & \multicolumn{3}{|c|}{ Sample } & Density $\left(\mathrm{kg} / \mathrm{m}^{3}\right)$ & \multicolumn{3}{|c|}{ Particle size $(\mu \mathrm{m})$} \\
\hline & Cement type & SSA $\left(\mathrm{m}^{2} / \mathrm{g}\right)$ & PCE ratio & & & average & std \\
\hline A & LHY-05381 (regular) & 0.8 & - & 3130 & & 24 & 21 \\
\hline B & LHY-05381 (regular) & 0.8 & $0.12 \%$ & 3130 & & 24 & 21 \\
\hline $\mathrm{C}$ & LHY-04190 (fine) & 4.5 & $0.44 \%$ & 3130 & & 7.1 & 5.1 \\
\hline \multicolumn{4}{|c|}{ Silica type } & & range & average & std \\
\hline a & SiLiBeads 5210 & & & 2500 & $0-50$ & 28 & 12 \\
\hline $\mathrm{b}$ & SiLiBeads 5209 & & & 2500 & $0-20$ & 3.4 & 2.8 \\
\hline
\end{tabular}

Table 1 Characteristics of the solid materials (silica beads, cement) used in our experiments. The cement particle size has been obtained by laser granulometry and the surface specific area (SSA) by BET method. The ratio between superplasticizer (PCE) and cement weights is kept constant for a given sample, whatever the cement mass fraction $w$. The silica average particle size has been deduced from sieve analysis provided by producer.

stress $\sigma_{Y}$ that then reads $31 \mid 32$ :

$$
\sigma_{Y} \simeq \frac{225 \rho g \Omega^{2}}{4 \pi^{2} D_{f}^{5}}
$$

Here $\Omega=38 \mathrm{~mL}$ denotes the cone volume, $\rho$ is the fluid density and $g$ is the acceleration due to gravity. Note that Eq, 1 is accurate only when surface tension is negligible, i.e. for $D_{f}$ smaller than $\approx 20 \mathrm{~cm}$ or $\sigma_{Y}$ larger than $0.3 \mathrm{~Pa} 33$, which is not always verified here. However, as our study is mostly comparative, we will still use Eq1 1 even though this assumption is not always strictly valid. This method is also limited at high yield stress, when the fluid will not spread on a distance significantly larger than the bottom diameter of the cone. This corresponds to yield stresses larger than $500 \mathrm{~Pa}$ in our experimental conditions.

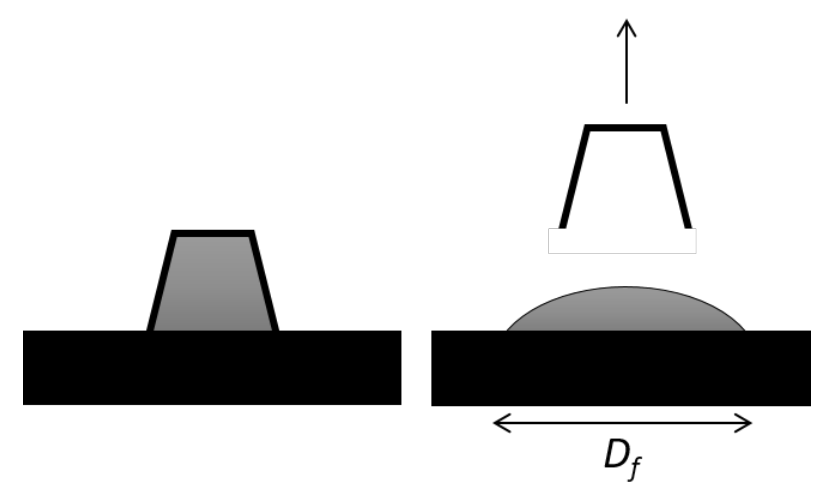

Fig. 1 Slump test used to measure the yield stress of the cement paste.

Figure 2 shows our yield stress measurements as a function of the particle mass fraction $w$ for the different systems considered. As expected, we observe that the paste yield stress $\sigma_{Y}$ increases with the solid fraction $w$, as predicted by the Yodel model 34 and that it decreases upon addition of superplasticizer (going from samples A to B in figure 2a). Yield stress values without superplasticizer have the same orders of magnitude as those reported in the literature for cement pastes with anionic surfactants at concentrations much lower than the critical micellar concentration, around $1 \mathrm{~Pa}$ for $w=0.5 .14$ We also observe that both cement samples with superplasticizer (B and C) show comparable yield stresses at a fixed cement concentration.

\subsection{Experimental set-up}

The experimental setup is depicted in figure 3. A $25 \mathrm{~mL}$ volume of fresh paste is poured in a beaker placed on a translation stage. The stage can be moved vertically at a prescribed velocity. Above the paste, we fix a square frame (inner side length $L=16 \mathrm{~mm}$ ), with cylindrical sides (diameter $4 \mathrm{~mm}$ ) while the lower side is kept open. The frame is made of acrylic-base resin and is obtained by $3 \mathrm{D}$ printing. A camera (IDS UI) is placed in front of the frame for recording images (typical frequency 30-50 Hz), which are subsequently analysed with the software ImageJ. The experiment is made as follows: the frame is initially dipped in the paste by rising the beaker and we lower the translation stage in order to withdraw the frame from the fresh cement paste at a velocity $V$. In this study, two withdrawing velocities, $V=2.8 \mathrm{~mm} / \mathrm{s}$ and $V=28 \mathrm{~mm} / \mathrm{s}$ have been used. The withdrawing is stopped when the frame is about to leave the paste, so that the withdrawn foam film, if it exists, touches the bath on its lower side (figure 4). The film is recorded from the side until its bursting, which takes typically a few tens of second. For each sample, this operation is repeated approximately 10 times. Between each withdrawing event, the sample has been re-homogenized with a spatula for around 15 seconds in order to limit sedimentation of the cement particles in the beaker. In the following, we vary the solid cement fraction in the paste, the particle size and the pulling velocity to define and understand what are the best conditions to obtain a stable soap film. For more details, we report in Table 2 the various experimental conditions tested, and the number of experiments performed, one experiment corresponding to typically 10 withdrawing events.

\section{Results}

\subsection{First observations}

Depending on the experimental conditions, two different behaviors are observed. On the one hand, some withdrawing events lead to the formation of particle laden films (case (i)), which can last more than $10 \mathrm{~s}$ (and up to more than $200 \mathrm{~s}$ ) after the end of the withdrawing stage (figure $4 \mathrm{a}$ and supplementary movie 1 $\dagger$ ). On the other hand, in case (ii), a film cannot be created, i.e. breaks before the end of the opening or immediately after (less than $5 \mathrm{~s}$, and most often less than $1 \mathrm{~s}$ ). A typical image sequence is shown in figure 4p (see also supplementary movie $2 \dagger$ ). In the following, we therefore try to characterize which experimental conditions lead to the first or second situation. 


\begin{tabular}{|c|c|c|c|c|}
\hline Sample & $\begin{array}{c}V \\
(\mathrm{~mm} / \mathrm{s})\end{array}$ & $w$ & $\begin{array}{c}\text { number of } \\
\text { experiments }\end{array}$ & $\begin{array}{l}\text { number } \\
\text { of films }\end{array}$ \\
\hline \multirow{9}{*}{ A } & \multirow{5}{*}{28} & 0.50 & 3 & \\
\hline & & 0.55 & 2 & \\
\hline & & 0.60 & 2 & \\
\hline & & 0.65 & 3 & \\
\hline & & 0.70 & 2 & \\
\hline & \multirow{4}{*}{2.8} & 0.55 & 1 & \\
\hline & & 0.60 & 1 & \\
\hline & & 0.65 & 1 & \\
\hline & & 0.70 & 2 & \\
\hline \multirow{5}{*}{ B } & \multirow{5}{*}{28} & 0.50 & 2 & 10 \\
\hline & & 0.60 & 5 & 49 \\
\hline & & 0.65 & 3 & 29 \\
\hline & & 0.70 & 5 & 19 \\
\hline & & 0.75 & 4 & 4 \\
\hline \multirow{3}{*}{ C } & \multirow{3}{*}{28} & 0.55 & 1 & 7 \\
\hline & & 0.60 & 1 & 4 \\
\hline & & 0.65 & 1 & \\
\hline \multirow{7}{*}{$\mathrm{a}$} & \multirow{7}{*}{28} & 0.713 & 1 & \\
\hline & & 0.767 & 2 & \\
\hline & & 0.775 & 1 & \\
\hline & & 0.780 & 2 & \\
\hline & & 0.803 & 2 & \\
\hline & & 0.810 & 1 & \\
\hline & & $0.817^{*}$ & 2 & \\
\hline \multirow{13}{*}{$\mathrm{b}$} & \multirow{8}{*}{28} & 0.49 & 1 & 8 \\
\hline & & 0.59 & 1 & 11 \\
\hline & & 0.69 & 1 & 11 \\
\hline & & 0.71 & 1 & 9 \\
\hline & & 0.721 & 2 & \\
\hline & & 0.725 & 3 & \\
\hline & & 0.73 & 1 & 3 \\
\hline & & 0.77 & 1 & - \\
\hline & \multirow{5}{*}{2.8} & 0.49 & 1 & \\
\hline & & 0.77 & 1 & \\
\hline & & 0.79 & 1 & \\
\hline & & 0.81 & 1 & \\
\hline & & 0.83 & 1 & \\
\hline
\end{tabular}

Table 2 For each experimental condition (one sample, one solid fraction, one withdrawing velocity), we report the number of experiments performed. One experiment corresponds to approximately 10 withdrawing events. When filled, the last column indicates the number of films used to calculate the average film lifetimes reported in figure $8{ }^{*}$ At this high concentration, note that the suspension is not fluid anymore.
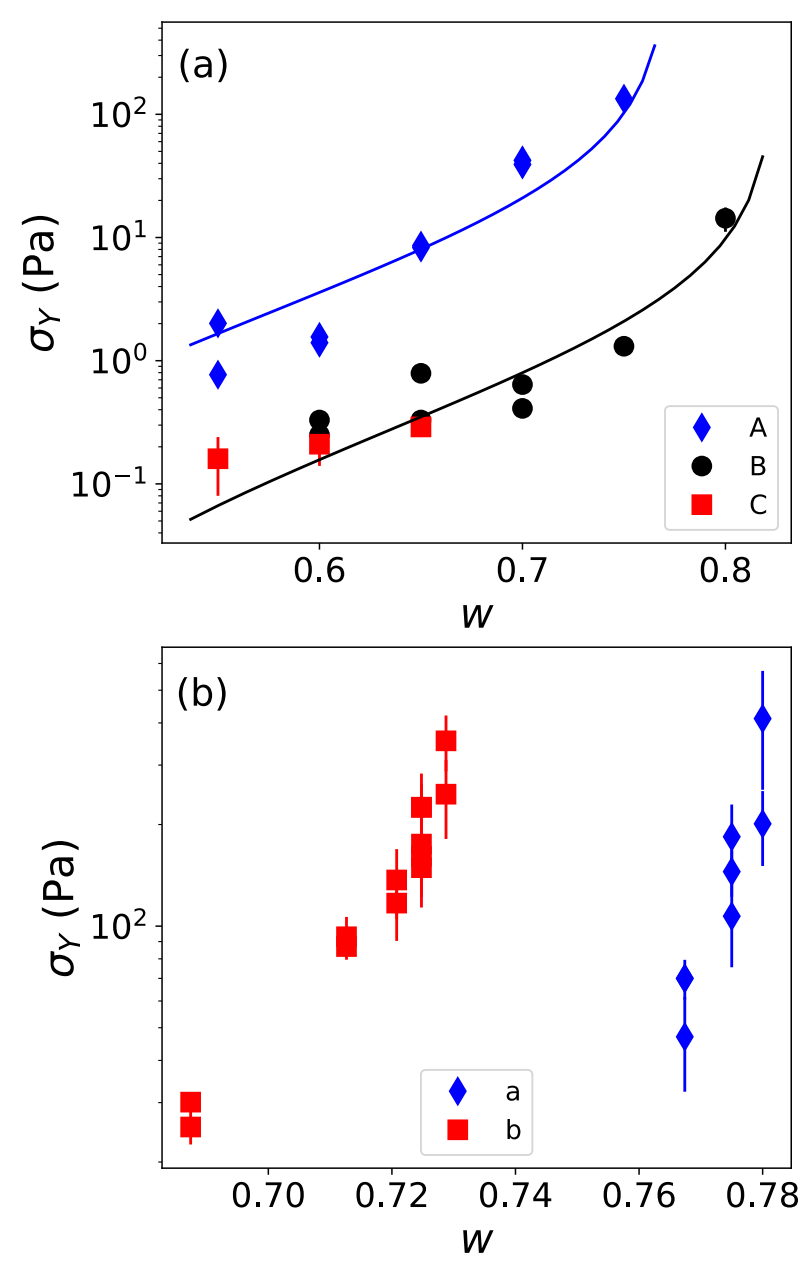

Fig. 2 Yield stress $\sigma_{Y}$ as a function of $w$. (a) Cement pastes: blue diamonds: sample A (regular cement, no PCE), black circles: sample $B$ (regular cement with PCE), red squares: sample $C$ (fine cement with PCE). (b) Silica suspensions: blue diamonds: sample a (particle size 0$50 \mu \mathrm{m}$ ), red squares: sample b (particle size 0-20 $\mu \mathrm{m}$ ). The solid lines are guides to the eye following the Yodel model 34 .

\subsection{Probability to make a cement foam film \\ 3.2.1 Influence of cement mass fraction}

In order to characterize the foam film formation from the cement suspension, we therefore calculate for each experiment the probability $P$ to make a foam film, defined as the number of films corresponding to case (i), divided by the number of withdrawing events (typically 10 in each experiment). We consider that a film is stable if it lasts more than 3 seconds for the cement suspensions, and 1 second for the silica suspensions. Indeed, even if they are almost always predictive, our experiments show in some conditions fluctuations of the outcome, which can be attributed to external factors such as mechanical vibrations of our set-up or internal ones, such as particle polydispersity or suspension heterogeneities. Error bars reflect the number of withdrawing events and experiments we performed in each case.

We first investigate the influence of the cement mass fraction $w$, at fixed velocity $V=28 \mathrm{~mm} / \mathrm{s}$. Figure 5 shows that, for all sample types, $P$ decreases with $w$. As the particle concentration is increased and the fresh cement becomes more and more pasty 


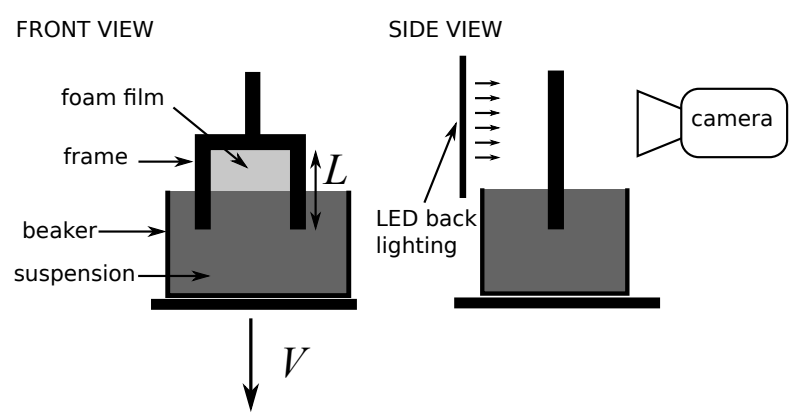

Fig. 3 Sketch of the experimental setup.

and viscous, it becomes more difficult to create a foam film. For instance, for sample A (regular cement LHY-05381, no superplasticizer), the probability to make a soap film drops abruptly from $P \approx 1$ to $P \approx 0$ for $w$ in the range $0.55-0.65$.

In cement-based applications, it is however crucial to ensure a minimum cement mass fraction in the fresh paste, as it is directly related to the mechanical properties of the final setted cementitious material. A natural direction to improve the possibility to form cement films at high cement fraction is then to incorporate a superplasticizer, which is expected to improve the paste workability, that is, to decrease the paste yield stress and apparent viscosity 30 .

Figure 5 therefore shows that the same qualitative behavior is observed when superplasticizer is incorporated - from samples A to B - but the drop in foamability $P$ is observed for a higher cement mass fraction, typically $w=0.7$ instead of $w=0.6$. This suggests that making the cement paste more fluid is a necessary step to improve foamability, here the possibility to make a cement film. In the following, we therefore quantify how this probability $P$ is influenced by the rheology of the fresh cement and silica pastes, via their yield stress $\sigma_{Y}$.

\subsubsection{Influence of yield stress and particle size}

Based on slump test measurements of the yield stress $\sigma_{Y}$ (section 2.2. we plot in figure 6 the probability to make a foam film $P$ as a function of $\sigma_{Y}$, for various sample types, for both cement and silica pastes. For each sample type, the yield stress is varied with the solid mass fraction.

As expected, we find that in all cases the probability $P$ drops when increasing $\sigma_{Y}$. Comparing cement samples A and B (respectively without or with superplasticizer), we also observe that this change of behaviour occurs at a larger yield stresses without superplasticizer (figure 6a): the transition occurs for $\sigma_{Y} \approx 0.5-1 \mathrm{~Pa}$ without superplasticizer (sample A), compared to $\sigma_{Y} \approx 2-10 \mathrm{~Pa}$ with superplasticizer (sample B). This suggests that the paste rheology is not the only parameter that determines the ability of the withdrawn cement to form a stable foam film.

Beyond their macroscopic effect on rheology, superplasticizers like PCE are known to drastically reduce the aggregation of the cement particles, by decreasing the particle-particle attraction through steric effects 30 . The effective grain (i.e. aggregate) size is therefore expected to be larger without the fluidizing additive. We therefore wonder what is the influence of the cement grain size on the cement apparent foamability.

To assess this point, we therefore conducted additional experiments with a finer cement LHY-04190 with superplasticizer (sample $\mathrm{C}$, see table 1). While the cement grain size has little influence on the paste yield stress (compare samples $B$ and $C$ in figure 2 ), the transition from $P \approx 1$ (cement films can be created) to $P \approx 0$ (no cement films) occurs for a much lower yield stress $\sigma_{Y} \approx 0.2-0.3 \mathrm{~Pa}$ for the finer cement (sample $\mathrm{C}$ ). This result confirms the expectations from the effect of superplasticizer: for a constant suspension yield stress the smaller the grain/aggregate size, the more difficult it is to create a cement foam film (figure 2 a). At this point, it should be mentioned that there are also variations in the chemistry of samples A, B and C that could explain the observed trend. However, the effect of the particle size is also observed for our simpler model system, the silica pastes, for which the solution chemistry is the same for all samples. For $\sigma_{Y} \sim 200 \mathrm{~Pa}$, we find that $P=1$ for particle size in the range $0-50 \mu \mathrm{m}$ (sample a), while it drops to $P \approx 0.3$ for finer particles (sample b, range $0-20 \mu \mathrm{m}$ ).

\subsubsection{Influence of withdrawing velocity: particle capture}

As we are interested in the role of dynamic conditions, we now investigate how the withdrawing velocity modifies the probability to make pasty soap films. We performed experiments with similar conditions (same setup and samples), but reducing the withdrawing velocity by a factor of 10 . At the smaller velocity $V=2.8 \mathrm{~mm} / \mathrm{s}$, a new regime is observed: particle-free film can be created, as reported in the inset of figure 77. We observed this behavior for both cement sample A (regular cement LHY-05381, no superplasticizer) and silica sample b (0-20 $\mu \mathrm{m}$ particles), under some specific experimental conditions.

More precisely, for both samples, figure 7 shows the probability to make a cement or particle laden film $P_{C}$, as well as the probability $P$ to make a foam film, which may indifferently contain particles or not, as a function of the solid mass fraction $w$. $P_{C}=P$ means that all created films contain particles, while $P_{C}=0$ and $P=1$ means that we always draw particle-free films. For the larger velocity ( $V=28 \mathrm{~mm} / \mathrm{s})$, particles are present in almost all cases where a film is created. For the smaller velocity, we find that particle-laden films can be created for larger solid fractions $w$, and that particle-free films are still stable at even larger $w$.

\subsection{Lifetime of pasty soap films}

In order to elaborate a mineral foam with disconnected cells, cement paste foams have to be stable enough and not to collapse in order to enable setting to take place inside the cement laden films. This requires that the cement foam film lasts long enough. To quantify the cement film stability, we therefore measure the lifetime of the withdrawn cement laden films.In figure 8, we plot the average lifetime of pasty foam films $T$ (obtained at $V=28 \mathrm{~mm} / \mathrm{s}$ ) as a function of the solid mass fraction $w$ for two cement samples and one silica suspension.

The lifetime $T$ is defined as the time elapsed between the end of the drawing phase and the film bursting.

For the cement sample B (figure 83), we observe that $T$ significantly increases (it is multiplied by a factor close to 6) as $w$ 

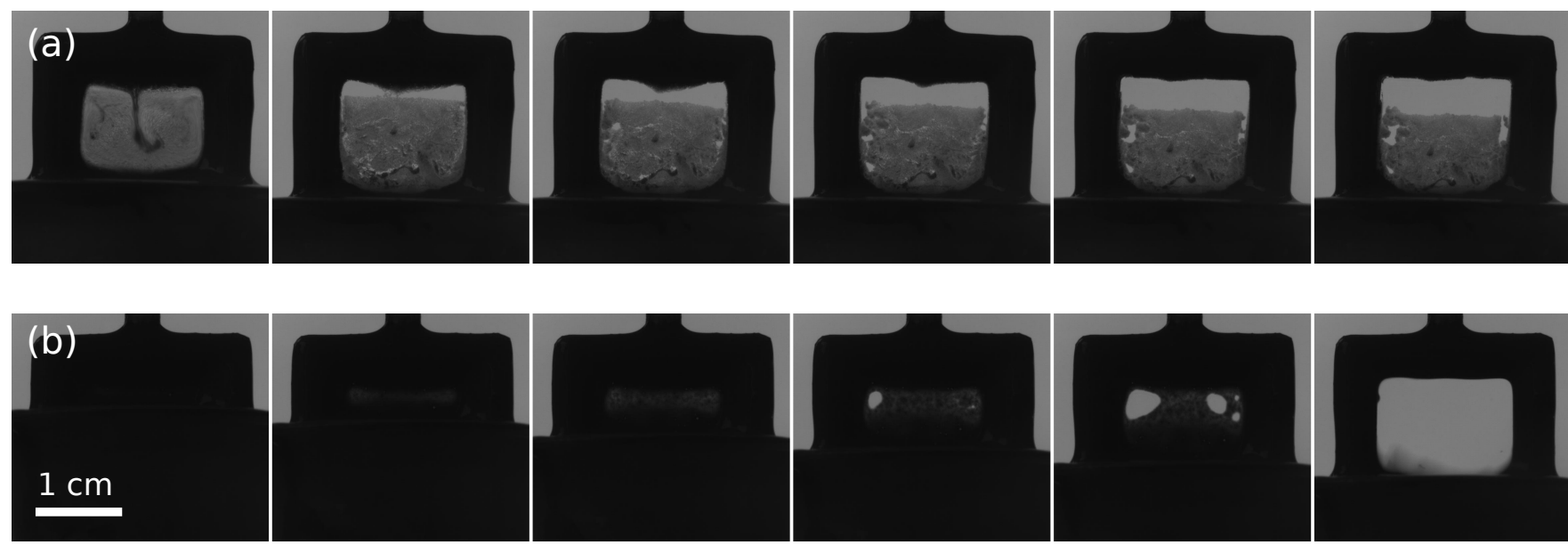

Fig. 4 Image sequences of two withdrawing events for $w=0.6$ (sample A, regular cement without PCE, $V=28 \mathrm{~mm} / \mathrm{s}$ ). (a) Case (i): Stable cement film (lifetime $18.7 \mathrm{~s}$ ). The timelapse between successive frames is $3.75 \mathrm{~s}$. (b) Case (ii): The withdrawn film breaks before the end of the frame pulling. The timelapse between successive frames is $0.05 \mathrm{~s}$, much shorter that in the first image sequence. See also corresponding movies as ESI $\dagger$.

increases from 0.5 to 0.75 . For the silica suspension (figure 8 p), we observe that the soap film lifetime goes through a maximum for $w \approx 0.6$. We discuss these seemingly antagonist behaviors in section 4 Finally, the film lifetime is also increased by a factor close to 3, at fixed solid fraction, when the particle size is decreased (from cement samples B to C, figure $8 \mathrm{~B}$ ).

It should also be noted that the longest lifetimes are measured at concentrations for which very few drawing events actually result into a stable film, as shown by the probability results reproduced in figure 8 . This suggests that for a given $V$-which could be fixed by the processing shear rates in a macroscopic foamthere exists an optimal cement concentration, which makes a compromise between the possibility to form cement laden films and their subsequent stability.

\section{Discussion}

\subsection{Capture of particles in the film}

We first discuss the ability for the created soap film to capture particles. This point is crucial for the applications in cementitious materials processing, as the presence of particles is a prerequisite to make solids with a closed porosity.

In particular, for a fixed cement concentration $w$, we find that particles can be captured only for the larger velocity (figure 7). This observation is reminiscent of experiments of dip-coating (supported films) of granular suspensions ${ }^{35136}$ which evidenced that only liquid and no particles were dragged below a threshold velocity.

A naive interpretation of this phenomenon is that for very viscous (or high yield stress) pastes, it is more favorable to suck water out of the paste than to actually deform it as a whole. The typical stress associated with the deformation of the paste is the yield stress $\sigma_{Y}$. Based on Darcy law, and assuming the paste permeability reads $\alpha d^{2}$, a typical stress to suck liquid out of the cement suspension scales as $\eta_{0} V /(\alpha d)$, with $\eta_{0}$ the dynamic viscosity of the interstitial solution. We will thus expect to get foam films containing cement particles for $\sigma_{Y} \ll\left(\eta_{0} V\right) /(\alpha d)$, that is, for low enough concentrations, high velocity or small particles. In this simple model, the effect of particle size is reminiscent of what is observed for the entrainment of suspension in dip-coating experiments in various geometries $35[37$.

In addition, we can estimate the velocity at which a transition is expected for the samples shown in figure 7. For cement sample A (cement without superplasticizer, figure $7 \mathrm{~B}$ ), we have $\sigma_{Y} \sim 10 \mathrm{~Pa}$ for $w=0.65$. We have no characterization of the aggregate size in our pastes without superplasticizer, but optical measurements in the literature ${ }^{\sqrt{38}}$ suggest it can be as large as five times the initial particle size, that is $d \sim 100 \mu \mathrm{m}$. From these and taking ${ }^{39} \alpha \sim$ $10^{-2}$, we expect a crossover from particle laden to particle free films for $V=V_{c}=\sigma_{Y} \alpha d / \eta_{0} \sim 1 \mathrm{~cm} / \mathrm{s}$, which is indeed compatible with the different behaviours evidenced for $V=28$ and $2.8 \mathrm{~mm} / \mathrm{s}$.

For the silica sample $\mathrm{b}$ at the lower velocity $V=2.8 \mathrm{~mm} / \mathrm{s}$ (figure $7 \mathrm{p}$ ), we also observe that the particles are no longer captured when $w \approx 0.77-0.79$. The estimated critical stress $\sigma_{c} \sim \eta_{0} V /(\alpha d)$ is $100 \mathrm{~Pa}$ (taking $d \approx 3 \mu \mathrm{m}$ ), of the same order of magnitude as the yield stress values (figure $2 \mathrm{p}$ ).

\subsection{Estimation of the thickness $h$ of the film}

We have found in section 3.2 .2 that the cement grain size $d$ modifies the ability to withdraw a cement film from the paste. More precisely, we find that reducing the particle size lowers the probability to form a film for a fixed suspension yield stress $\sigma_{Y}$. It would therefore be useful to compare $d$ to a natural lengthscale in this film drawing problem, which is the thickness $h$ of the created film.

\subsubsection{Prediction by continuum mechanics versus particle size}

Starting from the pioneering work of Mysels ${ }^{40}$ and up to recently $\frac{11112}{12}$, a lot of efforts have been devoted to the case of (particle-free) soap film withdrawing. From a modeling point of view, it was first proposed ${ }^{41}$ that the thickness of the film is selected by a balance between viscous forces and surface tension: $h \sim a \mathrm{Ca}^{2 / 3}$, where $a=\sqrt{\gamma / \rho g}$ is the capillary length, which compares gravity to surface tension effects and $\mathrm{Ca}=\eta V / \gamma$ with $\gamma$ the 

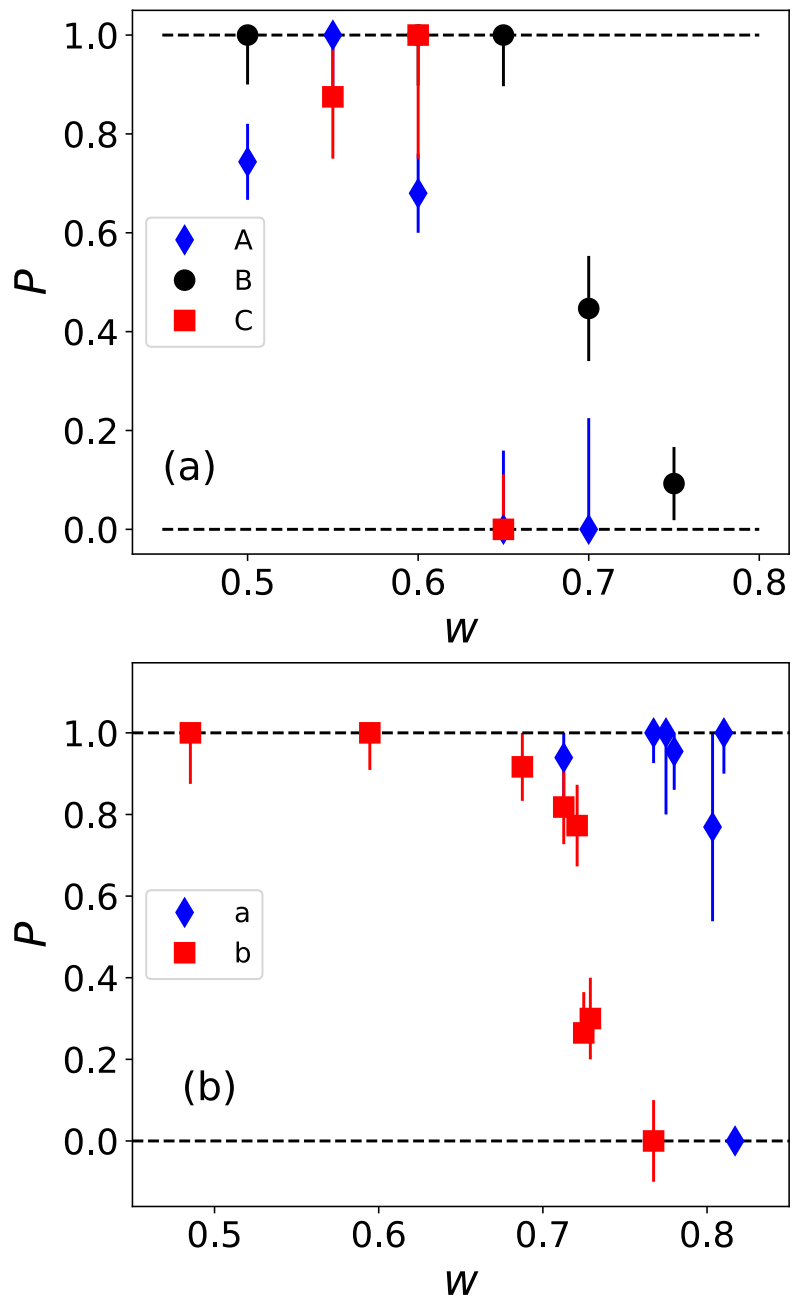

Fig. 5 Probability to create a foam film $P$ as a function of the solid mass fraction $w$ for $V=28 \mathrm{~mm} / \mathrm{s}$. (a) Cement pastes: blue diamonds: sample A (regular cement, no PCE), black circles: sample B (regular cement with PCE), red squares: sample $C$ (fine cement with PCE). Each data point corresponds to averages over 1-5 different experiments, see Table 2 for details. (b) Silica suspensions: blue diamonds: sample a (particle size 0-50 $\mu \mathrm{m}$ ), red squares: sample b (particle size 0-20 $\mu \mathrm{m}$ ).

fluid surface tension and $\eta$ its viscosity. In more details, the selected thickness thus depends on the surfactant dynamics and its diffusion and availability in the thin film, but under appropriate conditions, these dependencies with withdrawing velocity and fluid viscosity are reasonable.

For yield stress fluids like cement paste, Derjaguin and Levi ${ }^{42}$ have shown that this expression can be modified in order to account for the actual viscous stress in the film, which is, in the limit of vanishing velocities, $\sigma_{Y}$ instead of $\eta V / h$. The modified prediction for the film thickness is therefore $h \sim a^{3}\left(\sigma_{Y} / \gamma\right)^{2}$ : the film thickness is expected to increase with the yield stress, i.e. in our experiments, with the solid mass fraction $w$. This law was put in default by experiments ${ }^{43 / 44}$ in similar yet different case of a plate pulling a film of a polymer microgel, due to elastic interactions between the plate and the fluid in the bath. However, in our free film configuration, these elastic interactions do not exist, and there is, to the best of our knowledge, no experimental verifica-
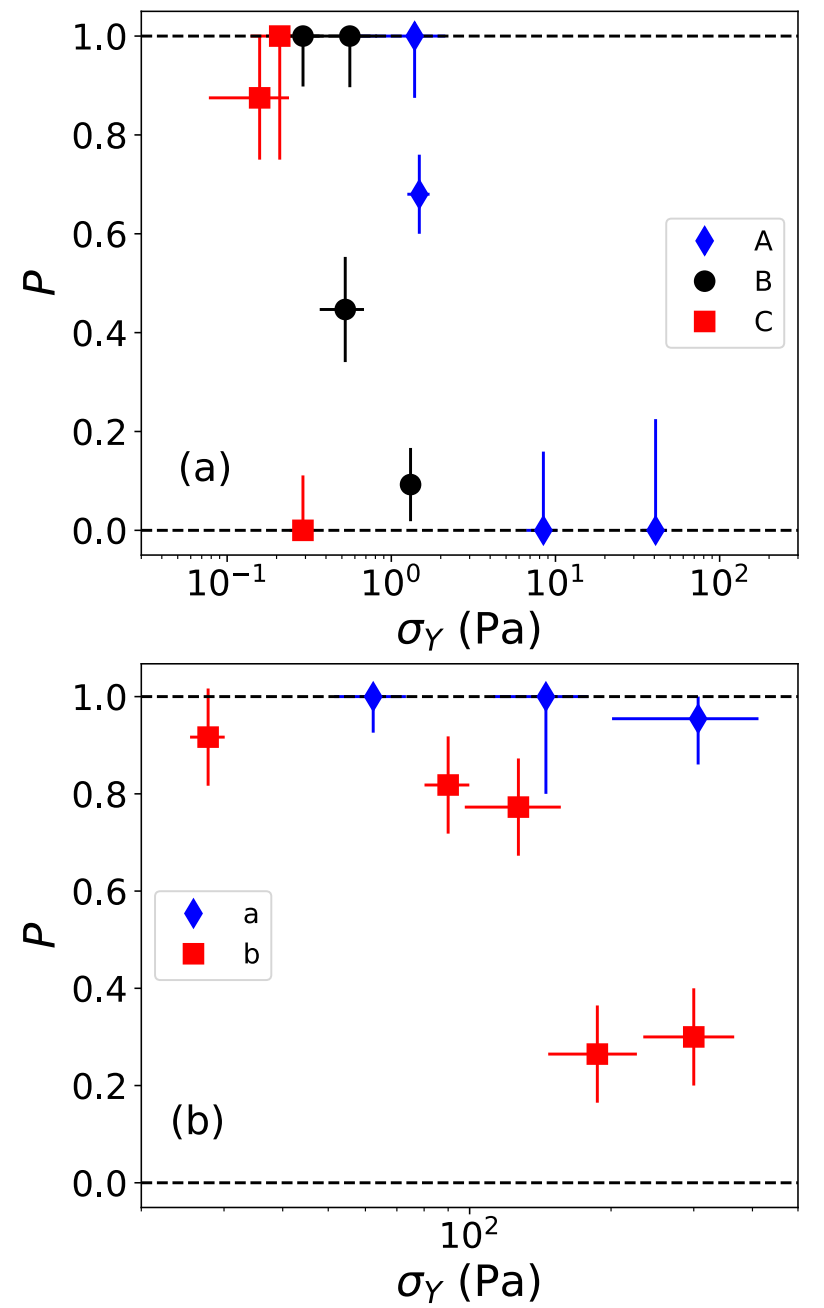

Fig. 6 Probability to create a foam film $P$ as a function of the yield stress $\sigma_{Y}$. (a) Cement pastes: blue diamonds: sample A (regular cement, no PCE), black circles: sample B (regular cement with PCE), red squares: sample C (fine cement with PCE). (b) Silica suspensions: blue diamonds: sample a (particle size 0-50 $\mu \mathrm{m}$ ), red squares: sample b (particle size 0$20 \mu \mathrm{m}$ ). The withdrawing velocity is fixed to $V=28 \mathrm{~mm} / \mathrm{s}$.

tion or contradiction of the prediction of Derjaguin and Levi. We therefore use it to estimate a typical film thickness.

In cement pastes containing Sodium Dodecyl Sulfate surfactant, very similar to the SDBS used in the present study, the surface tension at 0.1 times the critical micellar concentration was found close to $50 \mathrm{mN} / \mathrm{m}^{18}$. For sample B (regular cement LHY05381 with superplasticizer), the transition to no film creation occurs at $w \approx 0.7$, corresponding to a cement paste density of $1900 \mathrm{~kg} / \mathrm{m}^{3}$, which yields $a=1.6 \mathrm{~mm}$. For $\sigma_{Y}=1 \mathrm{~Pa}$, we therefore estimate $h \sim 2 \mu \mathrm{m}, 10$ times smaller than the average particle size $(24 \mu \mathrm{m})$. For sample $\mathrm{C}$ (fine cement), the transition occurs at $w \approx 0.63$ and $\sigma_{Y} \approx 0.25 \mathrm{~Pa}$, corresponding to $h \sim 0.12 \mu \mathrm{m}$, typically $1 / 60$ of the average particle size $7.1 \mu \mathrm{m}$. For the cement pastes, we therefore expect the prediction of Derjaguin and Levi, based on continuum equations, to fail in this case due to the granular character and the particle size of the fresh cement suspension.

In the slightly different geometry of dip-coating (film withdrawn from a bath by a solid plate), recent works ${ }^{35136}$ have 

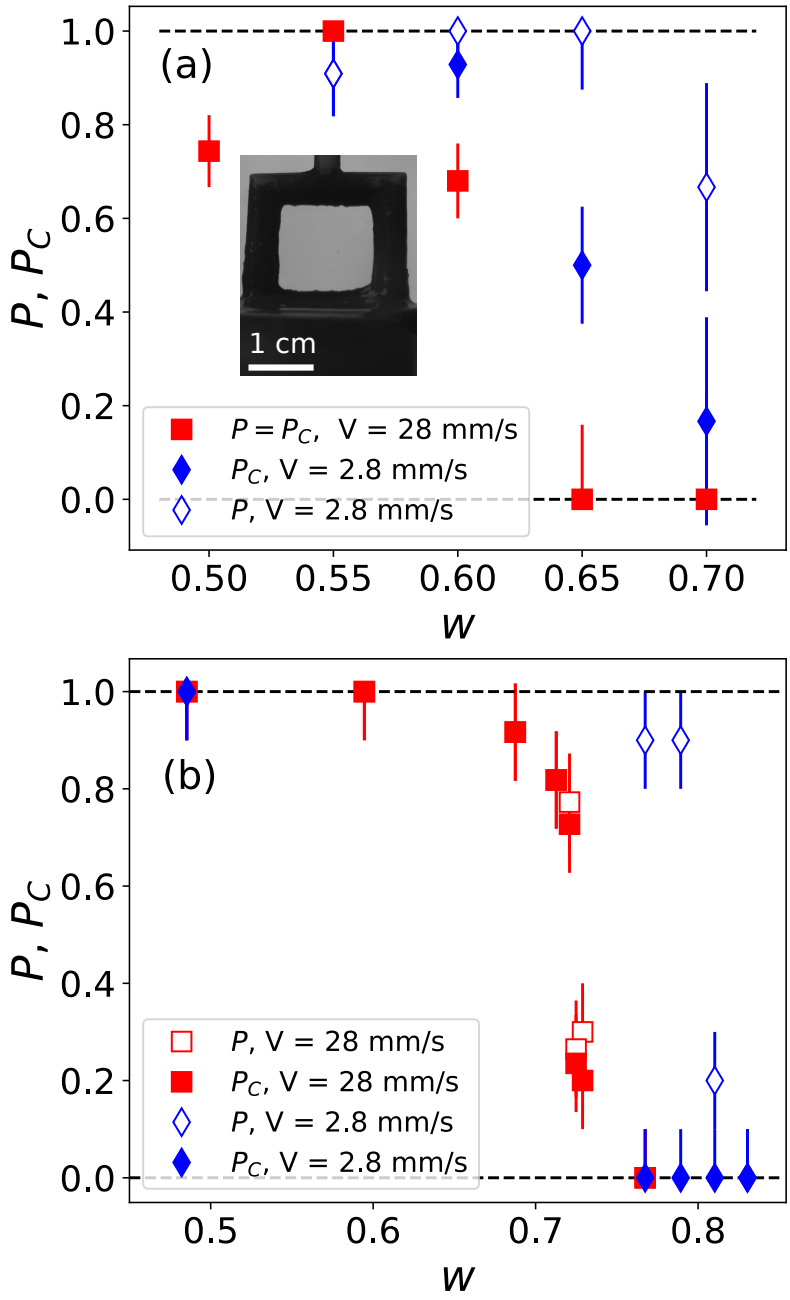

Fig. 7 Probabilities to make a cement or particle-laden film $P_{C}$ (solid symbols) and a foam film $P$ (empty symbols) as a function of $w$, for two different withdrawing velocities $V=2.8 \mathrm{~mm} / \mathrm{s}$ (blue diamonds) and $V=$ $28 \mathrm{~mm} / \mathrm{s}$ (red squares). A foam film may indifferently contain particles or not. (a) cement sample A (regular cement, no PCE), (b) silica sample b (particle size $0-20 \mu \mathrm{m}$ ). Inset: image of a particle-free soap film obtained for sample A at $w=0.7$ and $V=2.8 \mathrm{~mm} / \mathrm{s}$.

shown that, as the velocity and the viscous stress are decreased, the entrained film saturates to a thickness comparable to the particle diameter $d$. Similarly, we can expect the thickness of the entrained soapy cement film to scale as the typical particle size.

On the contrary, for the silica suspension of case $b$, the transition occurs at $w \approx 0.72$ and $\sigma_{Y} \sim 150 \mathrm{~Pa}$, yielding $h \sim 4 \mathrm{~cm}$ (with $\gamma \approx 60 \mathrm{mN} / \mathrm{m}$ the surface tension for the pure surfactant solution ${ }^{14}$ ). This is orders of magnitude larger than what is observed by naked eye on the sample (which actually form a film). This discrepancy could be explained by the effect of gravity, neglected in the thin film model of Derjaguin and Levi but which should play a role at large thicknesses.

\subsubsection{Light absorption by the cement films}

To compare the quantity of particles entrained, we measured for one sample the light intensity transmitted through the film for various solid fractions. The cement grains both absorb and scatter
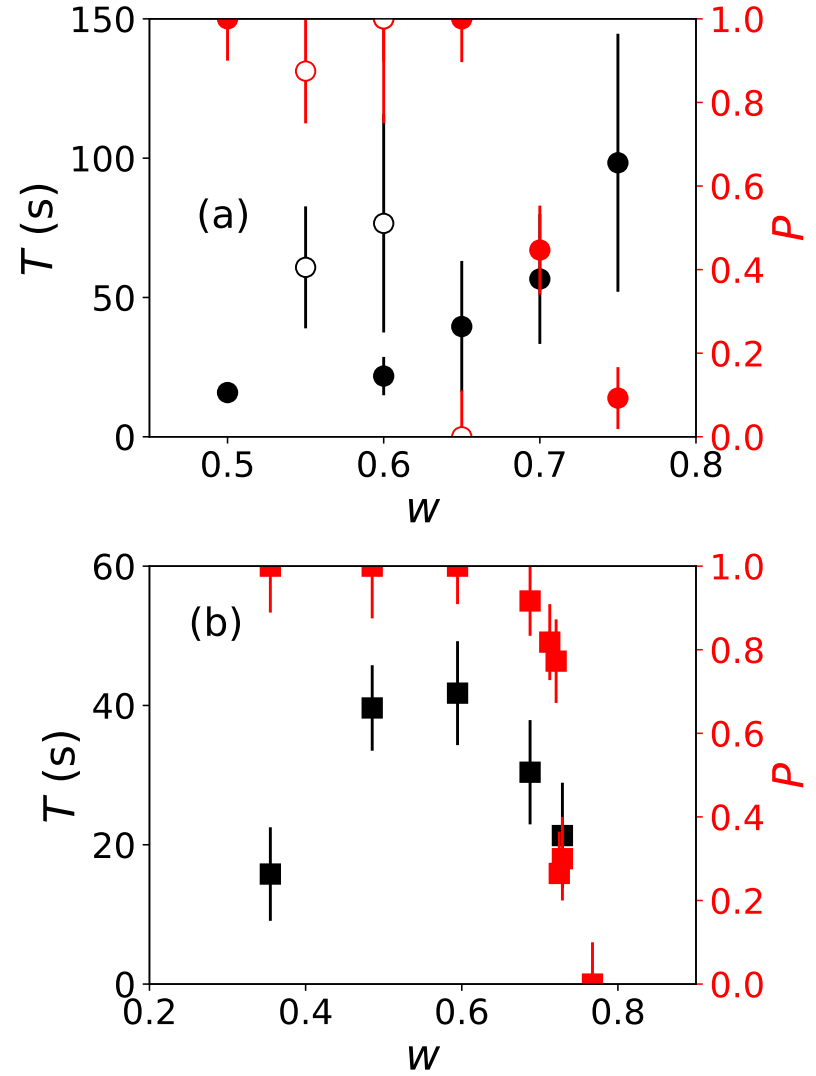

Fig. 8 Pasty film lifetime $T$ (black) and probability to make a film $P$ (red) as a function of the solid mass fraction $w$ : (a) cement samples $B$ (solid symbols, regular cement with PCE) and C (empty symbols, fine cement with PCE), (b) silica sample b (particle size 0-20 $\mu \mathrm{m}$ ). The withdrawing velocity is $V=28 \mathrm{~mm} / \mathrm{s}$. Each point corresponds to an average over 3-49 stable films, see Table 2 for the number of films considered. The error bars correspond to the standard deviation.

light, so that lower intensities indicate that more particles are entrained. We report in figure 9 the transmitted light intensity, normalized by the incident light, $I / I_{0}$ as a function of $w$, for cement sample B. We observe that the transmitted intensity decreases as the paste solid fraction and the velocity increase, indicating that more particles are captured. This suggests that the film thickness increases with the withdrawing velocity and the solid fraction, as expected since the viscous stresses get larger for both cases. However, extracting the thickness from the transmission coefficient would require a thorough optical calibration of the cement pastes, beyond the scope of the present study. Besides, it could also be modified by the local concentration of particles in the film, which may be different from the one in the bath. Discriminating between the two effects is unfortunately not possible with such images and would require independent measurements of the liquid and solid content, possibly through fluorescence measurements.

\subsection{Possible criteria for film generation}

To summarize, we find in our experiments that the probability to make a soap film $P$ decreases at large concentrations $w$, corresponding to large yield stresses of the suspension $\sigma_{Y}$. For a given 


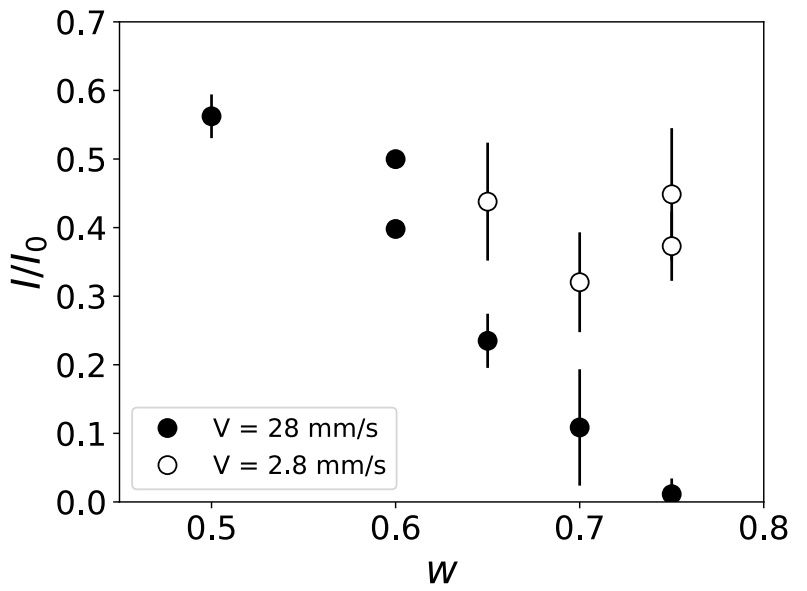

Fig. 9 Normalized transmitted intensity $I / I_{0}$ as a function of the solid mass fraction $w$ for sample B (regular cement with PCE) at two withdrawing velocities $V=2.8 \mathrm{~mm} / \mathrm{s}$ (empty symbols) and $V=28 \mathrm{~mm} / \mathrm{s}$ (solid symbols).

yield stress, an effect of the particle size $d$ is observed, large particles promoting film formation. We discuss here possible mechanisms which could set the stability of the freshly generated particle film.

For soap films of pure liquid, we expect the film to remain stable if its weight (per unit width) $\rho g h L$ does not exceed the surface tension variation in the film $\Delta \gamma$ at the origin of Marangoni stresses 23 . If we consider that continuum equations are still valid for our granular films, we can use the prediction of Derjaguin and Levi $h \sim a^{3} \sigma_{Y}^{2} / \gamma^{2}$ to predict that the film will break for $\sigma_{Y}^{2}>\gamma \Delta \gamma /(L a)$. Increasing the particle concentration increases the yield stress $\sigma_{Y}$ and destabilises the film. Taking $\gamma=50 \mathrm{mN} / \mathrm{m}$ and $\Delta \gamma=1 \mathrm{mN} / \mathrm{m}$ (typical value from the literature ${ }^{23}$ ), we find that the destabilisation should occur for $\sigma_{Y} \sim 2 \mathrm{~Pa}$. This result, which seems at first order in good agreement with our measurements on cement paste, does not explain many of our observations: the effect of the particle size and the larger yield stress for the silica suspensions.

Taking into account the granular nature of the suspension, and assuming that the entrained film thickness scales as $h \sim d$ as in dip-coating experiments ${ }^{35 \mid 36}$, we expect unstable films for larger particle size, which is incompatible with our observations.

Several ingredients are also likely to play a non-trivial role on the film stability. First, the role of the strong film-induced confinement on the paste rheology should be considered. Second, we expect the solid particles to play a surface-active role in our experiments, given the charge and concentrations of chosen surfactants. In this case, particle-stabilized monolayers are expected to be stable for capillary pressures up to $\gamma / d 4$. Smaller particles might therefore induce more resistant films. On the other hand, since we work at fixed SDBS surfactant concentration, finer particles could be less covered by the surfactant, hence less surfaceactive, since they have a larger specific area. Finally, the role of film inhomogeneities should also be investigated. We indeed observed that bare zones of the film (without cement particles), visible for instance in figure $4 \mathrm{p}$, are more prone to bursting, as reported in the literature in other configurations for model particulate systems $24 \mid 25$.

\subsection{Film stability}

We now discuss the stability of the fresh cement film, which can be characterized by its lifetime $T$, of the order of tens of seconds. For cement films, we found that $T$ increases with the solid fraction $w$. This could be due to the higher viscosity of the paste as $w$ increases, resulting in a slower drainage of the film.

On the other hand, for the silica films, we observe a maximum lifetime for an intermediate solid mass fraction $w \approx 0.6$. The decrease in lifetimes at high solid fractions could then be due to a lower solid fraction in the film, resulting in a less viscous film. We indeed observed that particle-free films could be generated at high solid fraction but the intermediate situation of a film poorly charged in particles may also be possible.

Finally, we also observe a slight increase of the film lifetime when the particle size is smaller, reminiscent of observations performed on expanding liquid sheets 25 . This point is attributed to the fact that large particles create large heterogeneities in film thicknesses, leading to particle bare zones of very unstable thin films 2425 . The influence of the particle size on the film lifetime could also be linked to the surface active role of the particles, as mentioned in the previous section.

\section{Conclusion}

In this work, we investigated the creation and stability of pasty foam films, created by withdrawing a rectangular frame from a bath of paste, which is either a fresh cement or a suspension of silica particles. This setup is a model experiment to study the dynamics of soap film creation, the elementary process of foaming. We have investigated the effect of the solid mass fraction, rheology modifier (superplasticizer), particle size and withdrawing velocity.

The first result is that cement films can only be created at low enough concentrations, i.e. for less viscous pastes. On the other hand, we found that the film stability is improved at high solid fraction, so that there exists an optimal concentration to ensure both foamability and foam stability, as also encountered in macroscopic 3D foams ${ }^{910}$.

Second, we evidenced that, beyond the paste rheology, the cement grain size plays a non-trivial role on the foamability: for a fixed suspension yield stress, large particles promote film creation.

Finally, we found that the withdrawing velocity also affects the ability to create a soap film, and its content (presence of particles). This suggests that the dynamics of the process of generating the foam might also modify the structure of the fresh liquid foam, and of the final solid foam (obtained after setting of the cement or silica sintering).

It would be interesting in the future to test to which extent these results could be applied to the elaboration of macroscopic 3D foams. In particular, in various foaming processes - direct foaming, or mixing of paste and foam - the foamy paste undergo deformations whose rate could affect the foam structure. 
At the film scale, this study also calls for an extensive characterisation of the entrained film (thickness and local concentration of particles), which could be envisaged through the combination of various optical methods like optical transmission and laser-induced fluorescence measurements.

\section{Acknowledgements}

This work was funded by the National Association of Research and Technology (ANRT, France) and the LafargeHolcim Innovation Center. We thank Véronique Schmitt, Rénal Backov, Martin Mosquet, Isabelle Javierre, Pierre-Henri Jézéquel and Pauline Petit for stimulating discussions. We thank Gilles Simon for his help for the experimental setup.

\section{References}

1 N. Narayanan and K. Ramamurthy, Cement and Concrete composites, 2000, 22, 321-329.

2 F. García-Moreno, Materials, 2016, 9, 85.

3 U. T. Gonzenbach, A. R. Studart, E. Tervoort and L. J. Gauckler, Journal of the American Ceramic Society, 2007, 90, 16-22.

4 I. Lesov, S. Tcholakova and N. Denkov, Rsc Advances, 2014, 4, 811-823.

5 E. Ebaretonbofa and J. R. G. Evans, Journal of Porous Materials, 2002, 9, 257-263.

6 J. Zhang, W. Liu, O. Gauthier, S. Sourice, P. Pilet, G. Rethore, K. Khairoun, J.-M. Bouler, F. Tancret and P. Weiss, Acta Biomaterialia, 2016, 31, 326-338.

7 K. Ramamurthy, E. Kunhanandan Nambiar and G. Indu Siva Ranjani, Cement and Concrete Composites, 2009, 31, 388-396.

8 N. Roussel, A. Lemaître, R. J. Flatt and P. Coussot, Cement and Concrete Research, 2010, 40, 77-84.

9 I. Lesov, S. Tcholakova, M. Kovadjieva, T. Saison, M. Lamblet and N. Denkov, Journal of Colloid and Interface Science, 2017, 504, 48-57.

10 B. Feneuil, N. Roussel and O. Pitois, Cement and Concrete Research, 2019, 120, 142-151.

11 L. Saulnier, F. Restagno, J. Delacotte, D. Langevin and E. Rio, Langmuir, 2011, 27, 13406-13409.

12 J. Seiwert, M. Monloubou, B. Dollet and I. Cantat, Phys. Rev. Lett., 2013, 111, 094501.

13 P. Petit, J. Seiwert, I. Cantat and A.-L. Biance, Journal of Fluid Mechanics, 2015, 763, 286-301.

14 B. Feneuil, O. Pitois and N. Roussel, Cement and Concrete Research, 2017, 100, 32-39.

15 U. T. Gonzenbach, A. R. Studart, E. Tervoort and L. J. Gauckler, Angewandte Chemie International Edition, 2006, 45, 35263530.

16 Z.-G. Cui, Y.-Z. Cui, C.-F. Cui, Z. Chen and B. P. Binks, Langmuir, 2010, 26, 12567-12574.

17 Q. Liu, S. Zhang, D. Sun and J. Xu, Colloids and Surfaces A: Physicochemical and Engineering Aspects, 2010, 355, 151-157.

18 P. Petit, I. Javierre, P.-H. Jézéquel and A.-L. Biance, Cement and Concrete Research, 2014, 60, 37-44.
19 L. Gibson and M. Ashby, Cellular Solids: Structure and Properties, Cambridge University Press, 1997.

20 R. Deleurence, C. Parneix and C. Monteux, Soft Matter, 2014 10, 7088-7095.

21 L. Champougny, B. Scheid, F. Restagno, J. Vermant and E. Rio, Soft matter, 2015, 11, 2758-2770.

22 Y. Timounay and F. Rouyer, Soft Matter, 2017, 13, 34493456.

23 L. Saulnier, L. Champougny, G. Bastien, F. Restagno, D. Langevin and E. Rio, Soft Matter, 2014, 10, 2899-2906.

24 Y. Timounay, E. Lorenceau and F. Rouyer, EPL (Europhysics Letters), 2015, 111, 26001.

25 P. S. Raux, A. Troger, P. Jop and A. Sauret, Physical Review Fluids, 2020, 5, 044004.

26 B. Jönsson, H. Wennerström, A. Nonat and B. Cabane, Langmuir, 2004, 20, 6702-6709.

27 J. W. Bullard, H. M. Jennings, R. A. Livingston, A. Nonat, G. W. Scherer, J. S. Schweitzer, K. L. Scrivener and J. J. Thomas, Cement and Concrete Research, 2011, 41, 12081223.

28 A. Zingg, F. Winnefeld, L. Holzer, J. Pakusch, S. Becker and L. Gauckler, Journal of Colloid and Interface Science, 2008, 323, 301-312.

29 D. Lowke and C. Gehlen, Cement and Concrete Research, 2017, 95, 195-204.

30 R. Flatt and I. Schober, Understanding the Rheology of Concrete, Woodhead Publishing, 2012, pp. 144-208.

31 N. Roussel, C. Stefani and R. Leroy, Cement and Concrete Research, 2005, 35, 817-822.

32 F. Dalas, S. Pourchet, A. Nonat, D. Rinaldi, S. Sabio and M. Mosquet, Cement and Concrete Research, 2015, 71, 115123.

33 R. J. Flatt, D. Larosa and N. Roussel, Cement and Concrete Research, 2006, 36, 99-109.

34 R. J. Flatt and P. Bowen, Journal of the American Ceramic Society, 2006, 89, 1244-1256.

35 A. Gans, E. Dressaire, B. Colnet, G. Saingier, M. Z. Bazant and A. Sauret, Soft Matter, 2019, 15, 252-261.

36 S. Palma and H. Lhuissier, Journal of Fluid Mechanics, 2019, 869, R3.

37 B. M. Dincau, E. Mai, Q. Magdelaine, J. A. Lee, M. Z. Bazant and A. Sauret, Journal of Fluid Mechanics, 2020, 903, A38.

38 J. H. Kim, H. J. Yim and R. D. Ferron, Journal of Rheology, 2016, 60, 695-704.

39 C. Cassar, M. Nicolas and O. Pouliquen, Phys. Fluids, 2005, 17, 103301.

40 K. J. Mysels and M. C. Cox, Journal of Colloid Science, 1962, 17, 136-145.

41 K. J. Mysels, K. Shinoda and S. Frankel, Soap films: studies of their thinning and a bibliography, Pergamon Press, 1959.

42 B. V. Derjaguin and S. M. Levi, Film Coating Theory, The Focal Press, London, 1964.

43 M. Maillard, J. Boujlel and P. Coussot, Phys. Rev. Lett., 2014, 112, 068304 
44 W. J. Smit, C. Kusina, J.-F. Joanny and A. Colin, Physical Review Letters, 2019, 123, 148002.

45 T. S. Horozov, Current Opinion in Colloid \& Interface Science, 2008, 13, 134-140. 